Pirineos, 149-150: 63 a 80, JACA; 1997

\title{
CARACTERIZACIÓN Y CONSECUENCIAS DE UN DESLIZAMIENTO EN UN ÁREA MARGINAL DEL PIRINEO ORIENTAL (CAVA, SIERRA DEL CADÍ, ENERO DE 1997)
}

\author{
DAVID MOLINA, JORDi NADAL \& JOAN MANUEl SORIANO
}

Departamento de Geografía. Universidad Autónoma de Barcelona, 08191 Bellaterra, Barcelona, España.

RESUMEN.- Las precipitaciones acaecidas entre noviembre de 1996 y enero de 1997 dieron lugar a un deslizamiento de tierras en las inmediaciones del pueblo de Cava, situado en la cuenca del río Segre (Prepirineo Catalán). La litología y la estructura de los materiales movilizados, asi como las prácticas de conservación agricola y su posterior abandono, han sido fundamentales en el desencadenamiento de este proceso erosivo. El estudio del fenómeno ha puesto de relieve dos partes diferenciadas: un movimiento rotacional en un sector de la ladera y una colada de barro originada por los materiales acumulados en la cabecera del torrente por el cual discurrió posteriormente. El análisis de las causas espacio-temporales de estos procesos y su interrelación con otros parecidos, así como la incidencia en las actividades humanas, son un factor esencial para definir estrategias de planificación y prevención.

SUMMARY.- The rain wich took place between november 1996 and january 1997 caused a landslide in the surrounding aren of the village of Cava, placed in the basin of the river Segre (Catalan Prepyrenees). The litology and structure of the mobilized materials, and the agrarian works and its further abandonement, as well, have been fundamental features causing the aforementioned erosive process. The study of this phenomena has highlighted the existence of two different parts: a rotational movement in a hillside's sector and a mud flow. The analysis of the space-time cause's of these processes, its inter-relation with similar ones, and its influence on the human activities, constitute an essential feature in order to define planification and prevention strategies.

RESUMÉ.- Les pluies ayant eu lieu entre novembre 1996 et janvier 1997 ont provoqué un glissement de terres aux alentours du village de Cava, situé dans le bassin de la rivière Segre (Prepyrenées de Catalogne). La lithologie et la structure des matériaux mobilisés, ainsi que les méthodes de conservation agricole et leur posté- 
PIRINEOS 149-150

rieur abandon, ont été fondamentaux pour le déclenchement de ce procés érosif. L'étude du phénomène a mis en relief deux parties differenciées: un mouvement rotational dans un secteur du versant et une coulée de boue originé par les matériaux accumulés dans la tête du ravin sur lequel elle s'est glissée plus tard. L'analyse des causes espacio-temporelles de ces processus et leur interrelation avec d'autres semblables, ainsi que leur incidence sur les activités humaines deviennent essentielles pour definir les stratégies de planification et prévention.

Palabras clave: dėslizamiento, movimiento rotacional, colada de barro, evento pluviométrico, abandono del territorio.

\section{Introducción}

Las abundantes y poco frecuentes precipitaciones que se prolongaron entre noviembre de 1996 y enero de 1997 en Cataluña han sido las causantes de un nuevo episodio de crecidas en la red hidrográfica. Las especiales características de estas precipitaciones explican en parte los movimientos de masa ocurridos durante los últimos días de este periodo en zonas próximas al proceso estudiado, entre las que cabe destacar el deslizamiento de $200 \mathrm{~m}$ lineales de talud que provocó la interrupción de la carretera N-260 entre Puigcerdà y La Seu d'Urgell por un período de más de dos meses y con el resultado de una costosa reparación.

Se considera interesante destacar uno de estos deslizamientos en una zona donde no había evidencias de grandes inestabilidades, pero que suponía un cierto peligro para la población de Cava, en la ladera norte de la Sierra del Cadí ( $\mathrm{N}$ de Cataluña). Por otro lado, la degradación de un medio en proceso de abandono, como son los campos de cultivo en las zonas de montaña, influye en el desencadenamiento de procesos erosivos donde intervienen parámetros fundamentalmente ligados a las características del substrato y a las condiciones meteorológicas, pero también a los tipos de usos del suelo y a sus cambios.

El análisis de sucesos como este es una herramienta fundamental para efectuar propuestas de planificación, sin embargo muchas veces esta relación necesaria entre los casos particulares y la ordenación del territorio no se traduce en un instrumento eficaz de prevención de catástrofes. Nuestro estudio pretende analizar las causas que provocaron un cambio fundamental en el paisaje y cómo pueden extrapolarse a otros lugares con vulnerabilidades similares. 


\section{Marco geográfico}

El curso alto del rio Segre divide al norte el Pirineo axial y al sur un conjunto de sierras que dan lugar a las mayores elevaciones del Prepirineo catalán, resultado de una compleja tectónica caracterizada por la superposición de diversos mantos de corrimiento. El sector más septentrional de los materiales secundarios y terciarios se da en el salto estructural de la Sierra del Cadí en su vertiente norte, que delimita de forma nítida un zócalo Paleozoico en el fondo del valle del río Segre y las primeras estribaciones de la sierra, y una transición hacia el Cenozoico en las partes más elevadas. No obstante, en un sector de contrafuertes se eleva un relieve, Tossal del Quer, que destaca por encima de otros, y que está constituido fundamentalmente por un bloque separado de la línea de riscos debido al sistema de fallas normales que acompañaron el levantamiento de la sierra. Las unidades superiores forman parte de la facies Garumniense, coronadas por un casquete de calizas con alveolinas del Eoceno inferior. A la altura del pueblo de Cava la base de estos materiales está formada por una barrera de calizas bioclásticas que dan paso a la discontinuidad con el Paleozoico.

Aunque la topografía general está gobernada por la línea de riscos del Cadí y los torrentes que descienden de ellos, en este sector de interfluvio, la vertiente está muy marcada por estas características litológico-estructurales. El cerro, Tossal del Quer, formado por rocas más duras da paso a unas pendientes más suaves sobre las arcillas garumnenses, que están mayoritariamente recubiertas por una potente cantidad de coluviones procedentes de las partes altas. En cotas inferiores el límite de la zona cultivable viene marcado por el risco calizo del pueblo, que se prolonga en un relieve de fuertes pendientes hasta el fondo del valle del río Cadí. En el sector de deslizamiento, la vertiente está atravesada por un torrente de curso temporal que ha favorecido un incremento y canalización de la pendiente, suficiente para la movilización de los materiales.

La vegetación que recubre el conjunto es mayoritariamente de tipo herbáceo o arbustivo, resultado de las sucesiones vegetales que han colonizado los antiguos campos de cultivo (mantenidos hasta 1950/60). En cualquier caso la cabecera está ocupada por una densa masa de Pinus sylvestris, al igual que el curso del torrente donde, además de árboles de ribera, también quedan importantes y centenarios conjuntos de Quercus pubescens (Figura 1 y Foto 1). 
$\mathbf{E}$
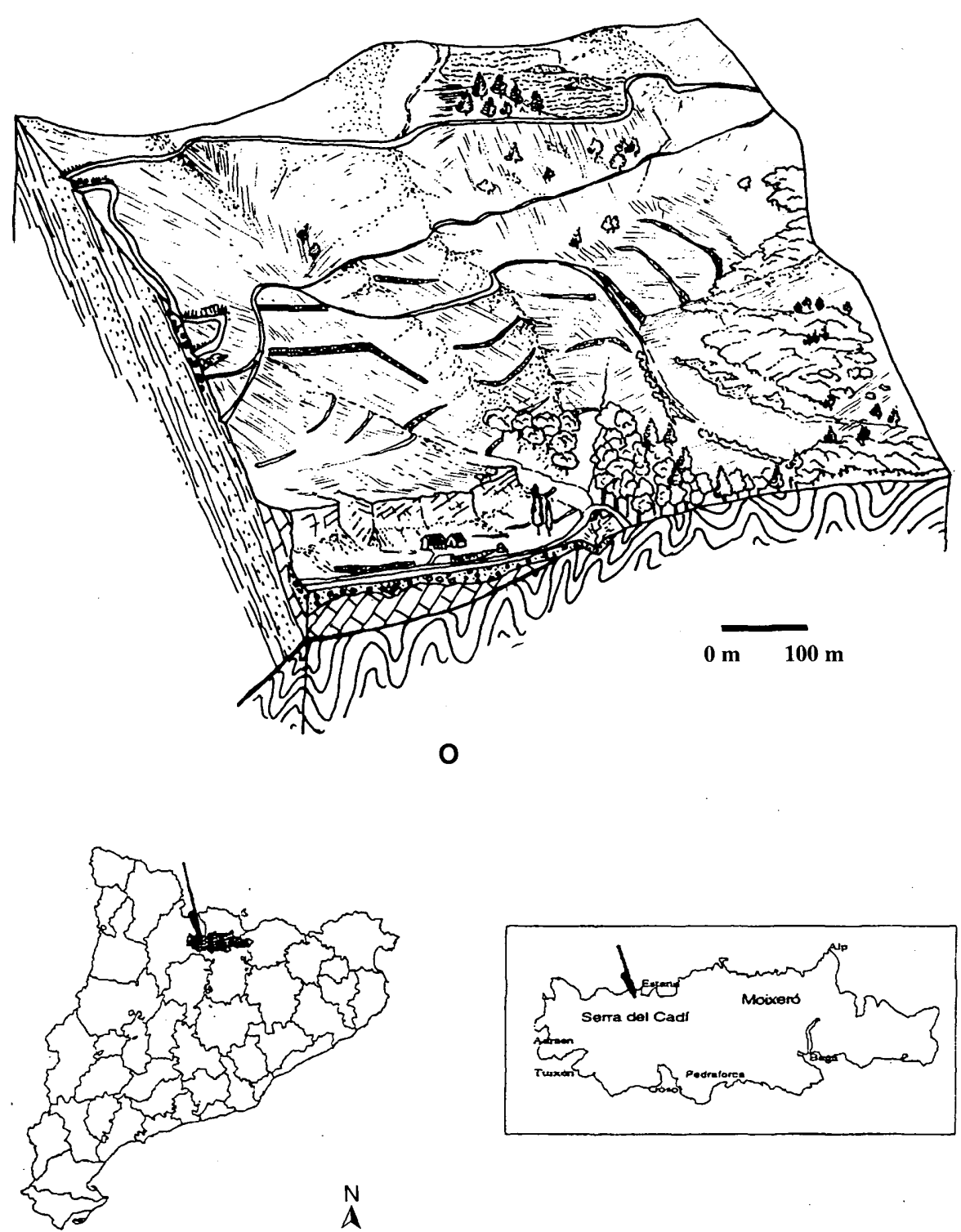

$\stackrel{N}{\Lambda}$

Figura 1. Situación previa al deslizamiento y mapa de situación en Cataluña y en el interior del Parque Natural del Cadí-Moixeró.

Previous landscape to landslide and situation maps.

66 
CARACTERIZACIÓN Y CONSECUENCIAS DE UN DESLIZAMIENTO...

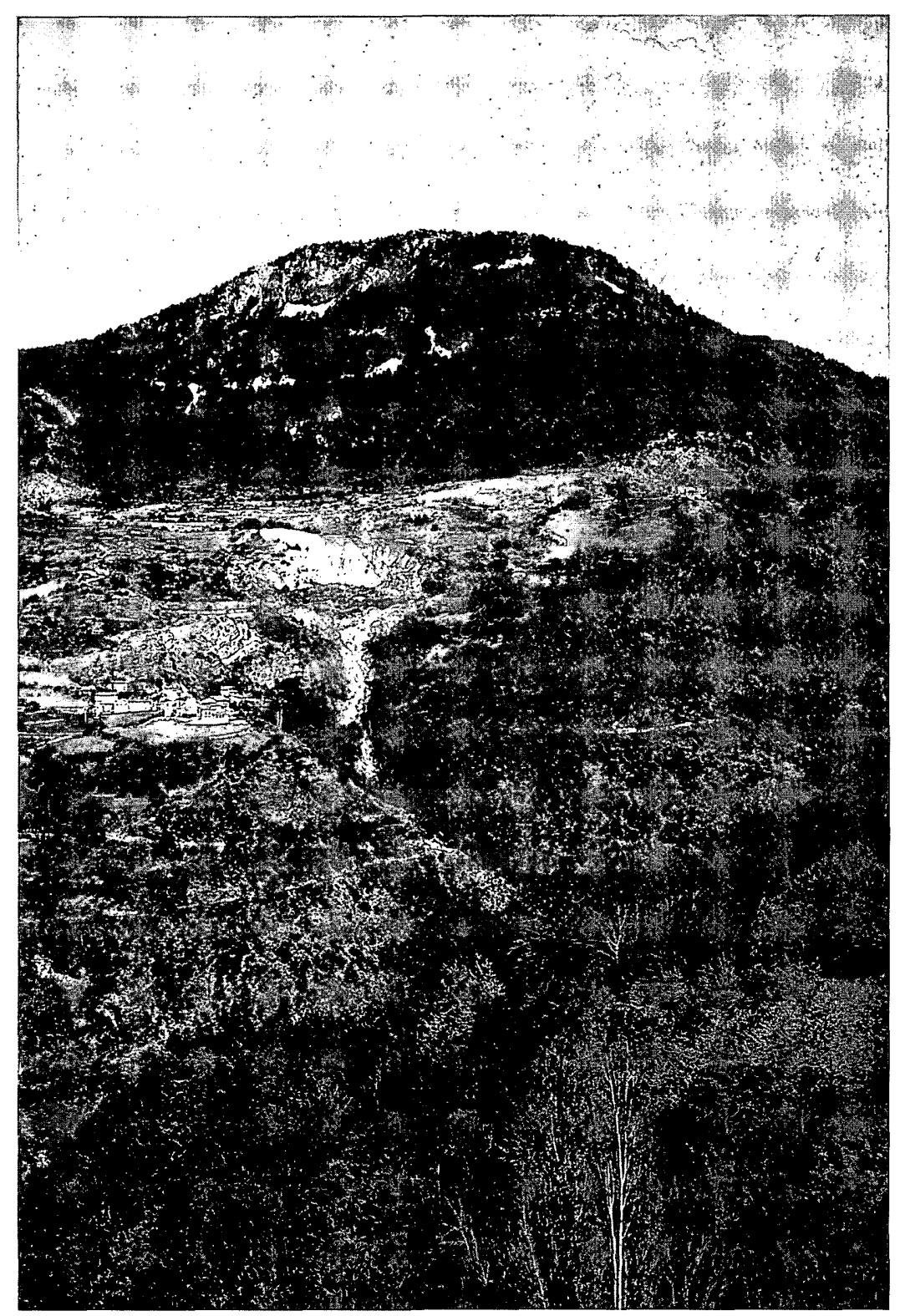

Foto 1. Imagen frontal del deslizamiento (vista hacia el este) donde se puede observar el movimiento rotacional y el inicio de la colada que separan a la izquierda el pueblo de Cava y en el extremo superior derecho de los campos, el barrio de Cal Pubill.

Landslide from west. Cava is under the cliff and Cal Pubill houses are at the right superior corner of fields. 


\section{Condiciones meteorológicas previas y estado del acuífero}

Las características de las precipitaciones que han dado lugar a este periodo de inestabilidades son bastante diferentes a otros periodos marcados por intensidades extraordinarias, como las lluvias de noviembre de 1982 (MARTÍN-VIDE, J. \& RASO, J. M.). Lo que ha sucedido en este caso ha sido provocado por una serie de bajas presiones que han favorecido la presencia de lluvias de baja intensidad pero ininterrumpidas durante varias semanas, con un descenso final de las temperaturas que ha convertido el agua en nieve, incluso en cotas relativamente bajas.

Entre el día 10 de noviembre de 1996 y el 22 de enero de 1997 se habían acumulado $340 \mathrm{~mm}$ de precipitación en la estación meteorológica de la Seu d'Urgell a $700 \mathrm{msnm}$ y $610,7 \mathrm{~mm}$ en La Molina a $1.500 \mathrm{msnm}^{1}$, a $10 \mathrm{~km}$ al oeste y $27 \mathrm{~km}$ al este respectivamente de la zona del deslizamiento y ambas en la vertiente norte de la Sierra del Cadí. En Cava, que se sitúa a $1.281 \mathrm{msnm}$, se habían acumulado durante el mes de enero de 1997 más de $20 \mathrm{~cm}$ de nieve que recubrían un suelo saturado de agua. Los aguaceros de los días 19, 20 y 22 de enero sumaron un total de $63 \mathrm{~mm}$ que acabaron por provocar la fusión total de la nieve caída con anterioridad en cotas inferiores a 1700-1800 m que en parte fueron a parar al sobresaturado acuífero superficial. No obstante, los caudales de base de ríos y torrentes eran anormalmente altos, así como los afloramientos de agua por multitud de puntos de intersección del acuífero, por ejemplo en la base de los márgenes de muchos campos de cultivo, como se podía observar el día 22 . No hay constancia de los caudales máximos ya que no existe ninguna estación de aforo en el río Cadí que drene el área afectada, pero es suficiente constatar la alarma hidrológica que se activó en la cuenca del río Segre y del Ebro en general, debido a estas circunstancias meteorológicas durante esos días.

La disposición de los materiales en la zona del deslizamiento hace que el acuífero se encuentre en la potente unidad de coluviones situados por encima de las arcillas rojas. La saturación de estos materiales con la consiguiente pérdida de cohesión entre ellos y la base del manto freático provocaron la fractura de cizalla que posiblemente originó el movimiento de masa. Las surgencias naturales de esta unidad se encuentran cerca de la confluencia de los dos torrentes que drenan la cuenca, debajo del risco de calizas cretácicas o en el contacto entre los materiales cuaternarios y el basamento arcilloso.

1 Datos climatológicos de las estaciones meteorológicas del Departament de Medi Ambient (La Seu d'Urgell) y del Instituto Nacional de Meteorología (La Molina). 


\section{Observaciones sobre el terreno y caracterización del proceso}

El proceso que se describe presenta dos morfologías bien diferenciadas determinando dos tipos de movimientos de masa, que se sucedieron en el tiempo el uno como consecuencia del otro. A continuación describimos las formas observadas sobre el terreno que indican las evidencias sobre este hecho y todos los elementos que pueden ayudar a reconstruir la génesis del deslizamiento.

\subsection{Primer proceso}

Situados en el tramo más bajo de la cuenca de recepción del torrente, antes de entrar en un marcado canal, el cambio de pendiente original que se encontraba en esta vertiente es el punto donde actualmente se sitúa la cicatriz de arranque del movimiento de masa a una cota de 1.495,28 m.s.n.m., que ocupa una longitud de 154,52 metros, con un salto entre el escarpe y la base de la fractura de 54,87 metros. El bloque desplazado se caracteriza por una forma general de tipo lobular, aunque este sector presenta numerosas grietas de distensión y rugosidades métricas y decamétricas que dan lugar a una superficie caótica. A pesar de esto, la disposición de la vegetación, suelo y materiales subyacentes mantiene una cierta estructura originaria, aunque muchos árboles han perdido la verticalidad. La disposición del bloque hundido responde perfectamente a un movimiento donde se ha producido una rotación de los materiales. Por otro lado, la cicatriz de arranque presenta un carácter marcadamente semicircular aunque como se puede observar en el diagrama correspondiente, hay una disimetría en las formas de los flancos. Este hecho se ajusta al relieve de la cuenca en esta zona donde se produce una abertura hacia el noroeste, lugar donde se han acumulado diversos cordones de materiales del lóbulo principal en forma de diques laterales, mientras que en el flanco opuesto la cicatriz de arranque se prolonga 136,95 metros (Figura 2).

El basculamiento y la importante acumulación de materiales en el frente de lóbulo principal ha provocado la aparición de una depresión al pie del talud de arranque, que ha favorecido la retención de agua.

La naturaleza de los materiales removidos tiene un componente mayoritario de gravas y bloques con una matriz terrígena, junto con un paquete edáfico importante, como refleja la granulometría de la tabla 1 . Así mismo, en el talud provocado por la fractura se observa que una parte de las margas rojas subyacentes también se pueden haber movilizado. Desde la zona de contacto de las arcillas y los coluviones, muy bien marcada por las surgencias del nivel 


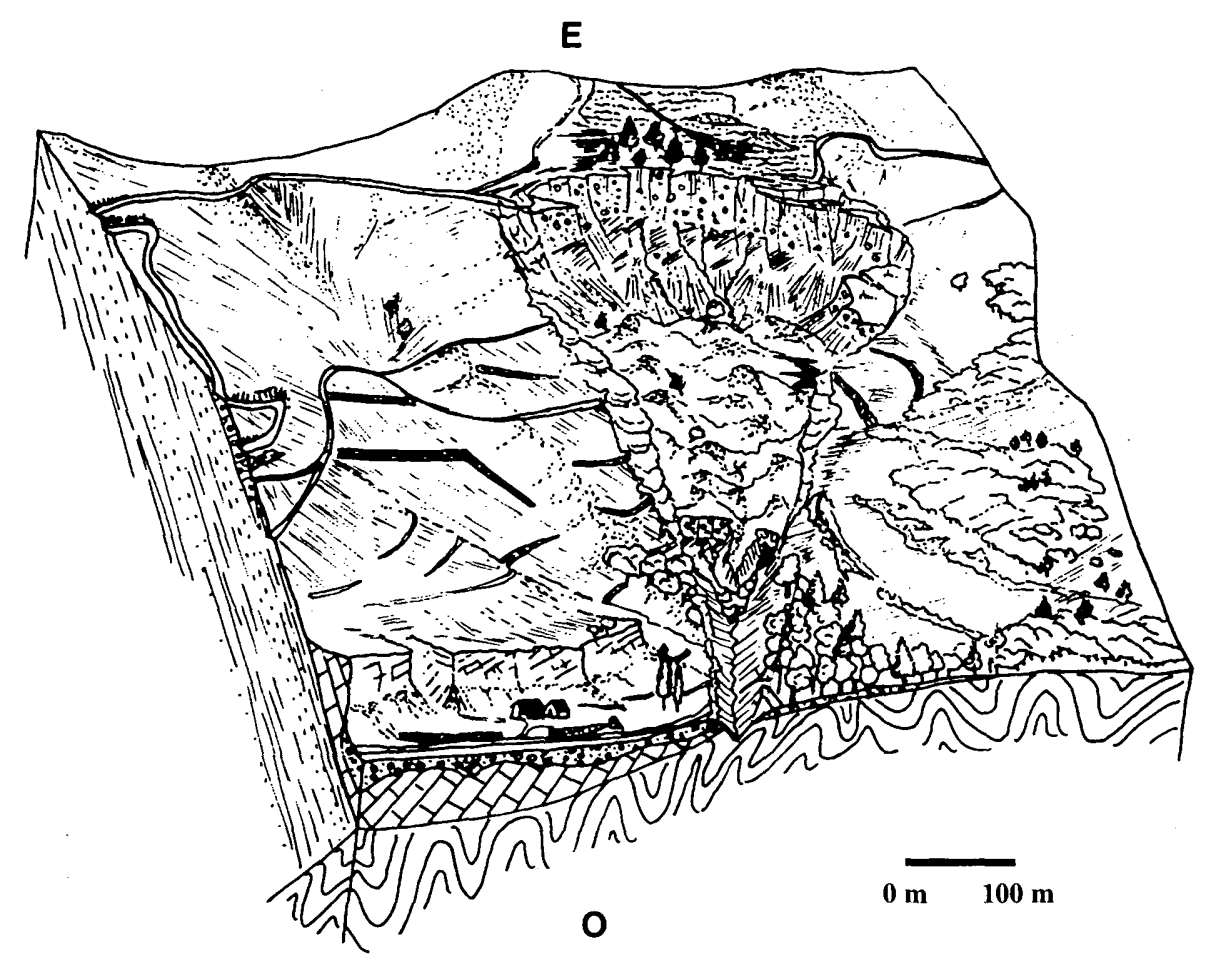

Figura 2. Bloque-diagrama del deslizamiento e inicio de la colada. Landslide geologic and topographic diagram.

\begin{tabular}{|lcc|}
\hline \multicolumn{3}{c|}{ Zona de inicio. Mass movement } \\
& $35,39 \%$ & $64,46 \%$ arcillas. Clay \\
& & $29,26 \%$ limos. Silt \\
& & $6,28 \%$ arenas. Sand \\
$>2 \mathrm{~mm}$ & $64,6 \%$ & gravas. Gravel \\
\hline Zona de desguace. End of mud flow, two months after \\
$<2 \mathrm{~mm}$ & $3,5 \%$ & $26,94 \%$ arcillas. Clay \\
& & $14,04 \%$ limos. Silt \\
& & $55,01 \%$ arenas. Sand \\
$>2 \mathrm{~mm}$ & $96,48 \%$ & gravas. Gravel. \\
\hline
\end{tabular}

Tabla 1. Granulometría de las muestras recogidas el 9-IV-1997. Textural analysis. 
de base de la capa freática, hasta la base del talud hay una altura de 35 metros que indican unos $168.000 \mathrm{~m}^{3}$ de materiales plásticos desplazados.

\subsection{Segundo proceso}

Las fuertes presiones que ejercen el peso de las gravas y bloques calizos sobresaturados de agua en el frente del lóbulo han provocado una mayor acumulación de agua y licuación de los materiales subyacentes, y debido al encajamiento de la masa en la zona inicial del canal de desagüe del torrente, estas presiones han aumentado en el interior de la masa.

La pérdida de la poca cohesión que mantienen los materiales removidos ha favorecido la aparición de una nueva cicatriz en el sector frontal de la masa con el inicio de un nuevo movimiento, en este caso de tipo fluidal tal como pone de manifiesto el aspecto que ha tomado el cauce de su recorrido y el frente de la colada al llegar al río Cadí, 707 metros más abajo del inicio de ésta.

Aquí los sedimentos presentan un carácter marcadamente lutítico con una plasticidad superior a los del movimiento anterior. Este hecho parece indicar que el arrastre del substrato arcilloso por parte de la masa hundida provocó una mayor acumulación de estos materiales en la parte inferior del lóbulo frontal, con una gran capacidad de convertirse en un colada. Si el deslizamiento ya fue bastante rápido (12-18 horas), la colada alcanzó velocidades de 10-15 m. $\mathrm{min}^{-1}$, hecho que le permitió recorrer todo el canal del torrente con una pendiente media de 20 grados y llegar al río principal, causando un cierto peligro de obturación de este, que si no llegó a producirse fue debido al poder erosivo de la crecida de aguas del río Cadí y a la naturaleza plástica de los materiales que se disolvieron al llegar al curso de agua.

A tenor de la velocidad que adquirieron los materiales y a su naturaleza podríamos clasificar la colada del tipo mud flow, hecho que no se refleja en el análisis de la muestra tomada en la zona inferior (Tabla 1), debido a que entre la finalización de la actividad de la colada y el día en que se recogió la muestra, pasó suficiente tiempo como para que los materiales mas finos fuesen lavados casi en su totalidad por las aguas de los dos cursos torrenciales.

$\mathrm{El}$ efecto erosivo de la colada ha profundizado un promedio de 9,65 m en el talweg del torrente y ha cambiado su morfología con la creación de unos diques laterales, sobre todo en el margen derecho, a causa de la expulsión lateral de los materiales fangosos más líquidos. En la parte baja del torrente, la acumulación de 2,5 metros de sedimentos en los campos situados sobre el cono de deyección, no han provocado daños debido a la fluidez de los materiales y a la disipación de la energía cinética de la masa al expansionarse, como puede observarse en algunos Populus nigra totalmente rodeados de sedimentos pero conservando la verticalidad. 
A partir de los datos del levantamiento topográfico, efectuado mediante GPS, de todo el movimiento y comparándolo con la topografía inicial, calculamos que se han movilizado $437.621 \mathrm{~m}^{3}$. El total de superficie ocupada es de $40.380,18 \mathrm{~m}^{2}$, con una densidad aproximada de $3,33 \mathrm{~kg} / \mathrm{l}$, calculándose un peso de los materiales de 1.457.278 Tm. (Figuras 3 y 4 ).

\section{5. ¿Qué fenómenos hicieron posible el suceso?}

A partir de las observaciones de campo y de la fotointerpretación, se han detectado formas antiguas de movimientos de masa, fundamentalmente en la parte alta de la ladera oeste del Tossal del Quer (Foto 1). Otros estudios referentes a la zona (CLOTET et al., 1985) no clasifican este área como zona de riesgo y por este motivo es doblemente interesante analizar cuáles han sido los factores que han provocado la superación del umbral de estabilidad de la vertiente.

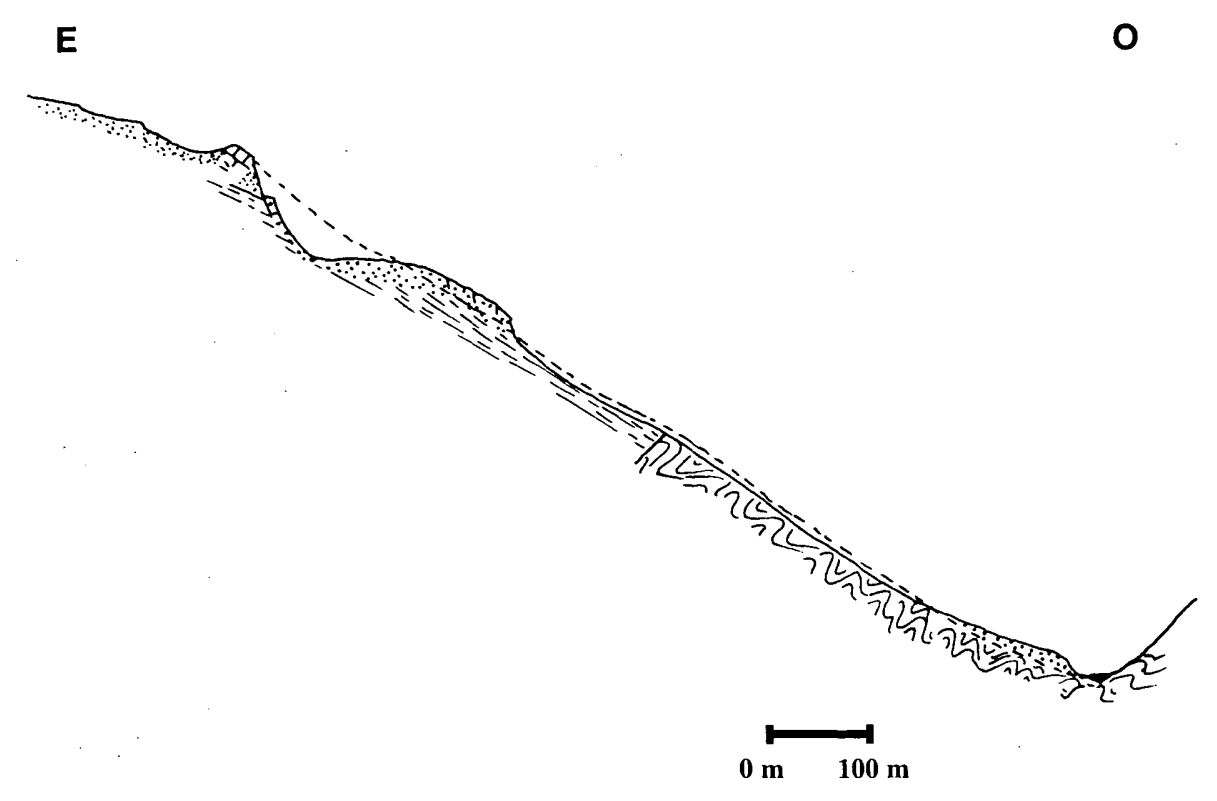

Figura 3. Esquema del deslizamiento. Landslide profile. 
Las abundantes precipitaciones que se sucedieron entre noviembre de 1996 y enero de 1997 favorecieron la saturación de las formaciones superficiales y una sobrecarga del acuífero del conjunto de coluviones. Las intensas precipitaciones y la fusión de la nieve los días 19, 20 y 22 de enero fueron el detonante de un exceso de peso en las formaciones superficiales que no podían drenar las aguas subterráneas a la misma velocidad que se producía la infiltración. Esta sobresaturación también incrementó la plasticidad de las arcillas sobre las que descansan los coluviones y que constituyen la base del acuífero, favoreciendo de esta manera una superficie de deslizamiento muy bien lubrificada sobre la que se desplazarían los materiales. La topografía del terreno también fue determinante para explicar la situación de la fractura inicial, que se ubica en un cambio de pendiente muy acusado entre una colina que separa un campo cultivado en un terreno mal drenado aguas arriba y una fuerte vertiente situada en la parte inferior. La existencia de este campo de cultivo de 1,28 hectáreas con un perfil cóncavo, favoreció un incremento de la presión hidrostática en este sector debido, por un lado, a la mayor acumulación del agua en el subsuelo y, por otro, a la presión de ésta en el talud de la ladera, dando lugar a la afectación parcial del campo $(0,23 \mathrm{ha})$.

La pendiente de la superficie de contacto entre arcillas y coluviones $15-20^{\circ}$ fue suficiente para facilitar el movimiento, pese a que la disposición de los materiales garumnenses se inclinan $50^{\circ}$ perpendicularmente a la vertiente. El afloramiento de las aguas freáticas en la fractura significó un incremento de agua en el bloque hundido, que a su vez, aumentaba el peso de éste. La porosidad de los materiales que constituyen el lóbulo principal, no retuvo esta nueva gran cantidad de líquido que inmediatamente pasó al plano de deslizamiento, con el agravante de que se había producido una importante acumulación de material arcilloso removido.

Hasta este momento el deslizamiento respondía a un hundimiento de ruptura plana rotacional típico con las cicatrices de distensión en la parte superior y la acumulación de tipo lobular en el frente de la masa. Las presiones del peso de ésta fueron las responsables del cordón lateral que aparece sobre todo en el flanco derecho. Por contra, las cicatrices y rugosidades internas del lóbulo responden al asentamiento de ésta sobre el terreno así como de la velocidad diferencial de la masa debida a la fricción en los flancos de ésta.

La llegada del frente de materiales a la parte alta del canal del torrente, donde la pendiente general del terreno pasa de $14^{\circ}$ a $31^{\circ}$, junto a la canalización que experimenta en este sector la ladera, favorecieron una nueva movilización de los materiales. Así mismo, lo que explica la diferente naturaleza de los sedimentos de la zona de arranque y de los que se encuentran en el río Cadí, al final del torrente, es la licuación de los materiales plásticos lubrificados, provocada por la gran presión en el sector frontal de la masa de materiales finos, gravas y 
agua y el encajonamiento del terreno. A partir de este momento se produce una nueva cicatriz en el frente del paquete que evacuó todos los sedimentos que dieron lugar a la colada de barro. La velocidad y capacidad de deslizamiento fue más elevada que en el movimiento anterior, debido por un lado a la viscosidad de los materiales, a la fuerte pendiente del terreno y a la saturación y circulación de agua superficial en el curso del torrente. La formación de diques laterales en este tramo resultó más acentuada en el margen derecho del curso de la colada que en el izquierdo, debido a la disimetría del terreno.

Por último el cese de la presiones sobre la cicatriz central implicó el fin del suministro de materiales a la colada que se interrumpió 3-4 días después de iniciarse. La gran plasticidad y licuación de los sedimentos fue la responsable de un vaciado casi absoluto del canal, que después se terminaría de limpiar con la circulación de las aguas subterráneas que afloraban en las dos cicatrices torrente arriba. Todos estos fangos, una vez acumulados en los campos situados en el cono de deyección, se aproximaron al curso del río Cadí aunque no llegaron a obturarlo.

\section{Incidencia del los usos del suelo en el desencadenamiento del proceso}

Generalmente en la descripción y análisis de procesos similares se incide fundamentalmente en las características geológicas del terreno y en las características del evento pluviométrico, dándose una menor relevancia a los elementos implicados en los movimientos de masa que se encuentran en la superficie del terreno o incluso en el conjunto edáfico. Por este motivo nos gustaría profundizar sobre lo que parece un elemento recurrente de los procesos acaecidos en los diversos eventos de los que se tiene referencia (GARCÍA-RUIZ \& PUIGDEFÁBREGAS, 1984; GARCÍA RUIZ \& LASANTA, 1994).

Muchos de los deslizamientos de gran magnitud y una inmensa mayoría de procesos rotacionales de pequeñas dimensiones tienen una relación directa con zonas de vertientes pronunciadas y que son o han sido cultivadas en algún momento histórico (GALLART et al. 1983; SEOTERS, 1984; COROMINAS, 1984; MOLINA, 1996) por el hecho de situarse en litologías blandas que generalmente dan lugar a suelos aptos para la agricultura así como un relieve modificado que presenta una mayor inestabilidad frente a los movimientos del agua en el suelo, aunque haya otros elementos a tener en cuenta. En nuestro caso existe un denominador común entre los materiales arcillosos del Garumnense que se encuentran ampliamente distribuidos en este sector del Pirineo y los grandes deslizamientos que se han producido his- 
tóricamente en ellos modificando un paisaje fundamentalmente agrario (CLOTET \& GALLART, 1983).

En el caso de Cava los cultivos de la ladera afectada tienen antecedentes como mínimo medievales, pero no todo el terreno que se ha desprendido era de cultivo ya que en la parte más elevada había una fuerte pendiente que seguramente se utilizaba como pastizal. En este tramo el camino recibe el nombre de Camí Nou (Camino Nuevo), debido a las correcciones que secularmente ha sido necesario efectuar en su trazado para evitar las grietas y demás modificaciones de la topografía que han aparecido.

El importante conjunto de campos de cultivo que hay por encima de la cicatriz, actualmente están abandonados, aunque hasta bien entrada la década de los años setenta se roturaron y sembraron. Su posterior uso como pastizales extensivos ha favorecido la desaparición de toda la red de drenaje antrópica que impedía el efecto pernicioso de la escorrentía concentrada encima de los márgenes ${ }^{2}$. Esta red, bien conservada mientras ha durado la actividad agraria, evacuaba con mayor celeridad el exceso de agua que se acumulaba en los campos (VIDAL, 1984), los cuales al ser cultivados tenían unos coeficientes de escorrentía superficial superiores a los actuales. Otros elementos que posiblemente han favorecido una mayor implicación han sido el cambio de vegetación en la cabecera de la cuenca, donde se ha pasado de un bosque muy poco denso con matorral, debido al pasto de mediados del siglo pasado, a un bosque denso de Pinus sylvestris que invade los campos situados a cotas superiores en la actualidad (Foto 1). El efecto de intercepción y absorción de la precipitación por las masas boscosas es evidente en lluvias débiles o moderadas, pero tiene poca importancia en periodos largos de precipitación o lluvias de mucha intensidad (GARCÍA RUIZ et al., 1996). En cambio, la retención de humedad en bosques cerrados con una orientación nada propicia a elevados índices de radiación solar y la facilidad de infiltración a partir de los sistemas reticulares, favorecen el mantenimiento y alimentación de las aguas edáficas y freáticas en eventos pluviométricos como el que aquí se trata.

Un buen ejemplo de la desestructuración de la red de drenaje que se organizaba en torno a los campos ha sido la erosión por escorrentía superficial concentrada del campo cultivado situado por encima de la fractura del deslizamiento. En este punto han coincidido otros elementos, como la forma cóncava y mal drenada de esta parte por causas naturales y también antrópicas, ya que en la actualidad no se presta la misma atención a la conservación del medio agrario de montaña que en el pasado.

2 La toponimia de los campos también ayuda a entender mejor la inestabilidad tradicional de este sector. Así, uno de los de mayores dimensiones arrastrados por el deslizamiento se llama "del Perilló" (del Peligro). 
Cava, Sierra del Cadí, Pirineos Orientales
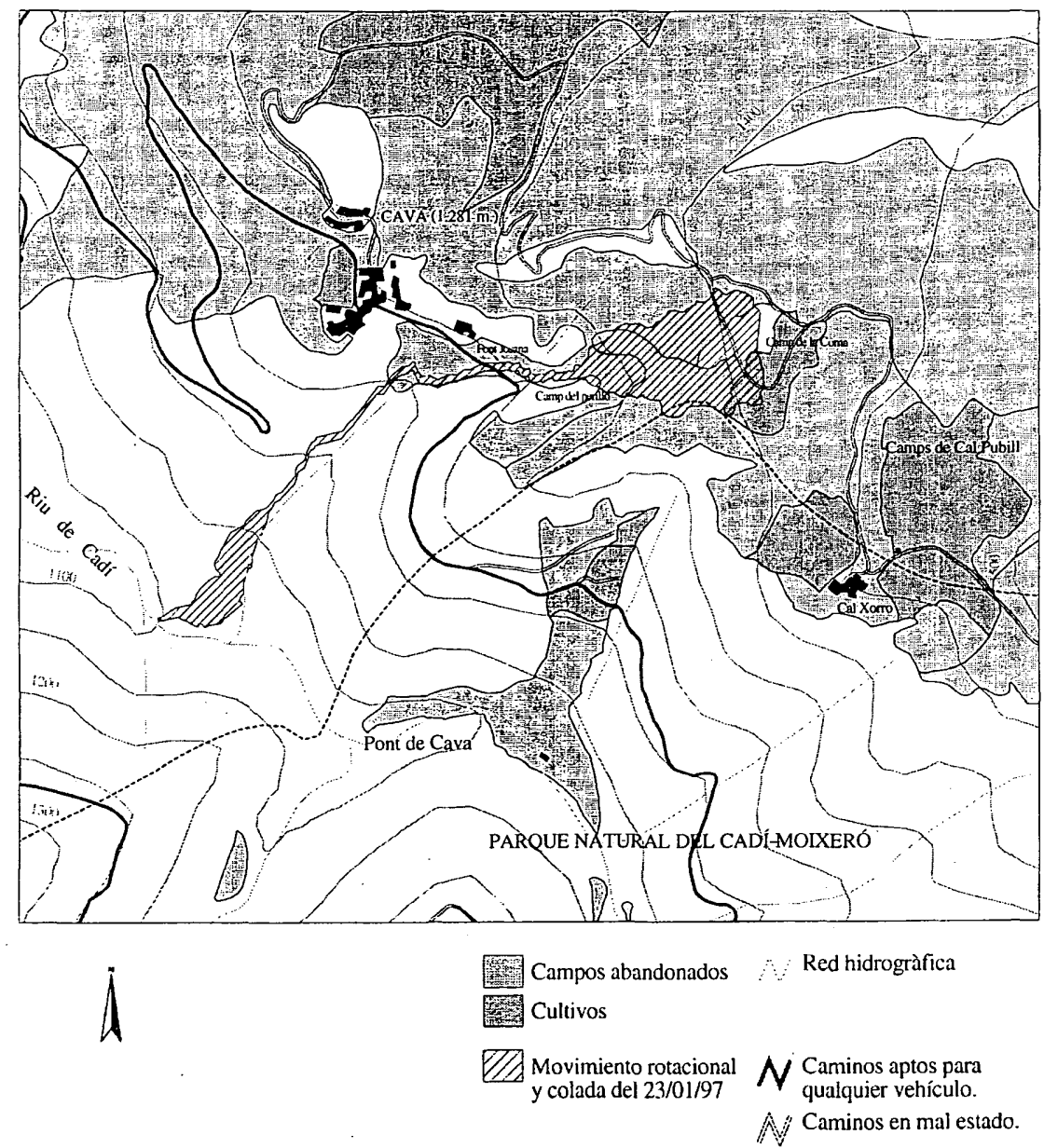

ESCALA 1:8.000

FUENTES: Elaboración propia a partir de los ortofotomapas 1:5.000 y delimitación mediante G.P.S. Equidistuncia de las curvas de nivel: 50 metros

Figura 4. Zona afectada por el desprendimiento. Landslide area and Cava village, abandoned and cultivated fields and roads.

76 
Parece difícil considerar la climatología y la naturaleza del substrato como los elementos únicos que han provocado la inestabilidad de la ladera, ya que situaciones similares se han podido dar en el pasado. En cualquier caso la predicción de estos fenómenos catastróficos de gran magnitud es muy difícil, ya que una.vez producidos no es fácil que se vuelvan a reproducir en el mismo sitio, aunque posiblemente sí en áreas similares. No hay que subestimar la capacidad de intervención del hombre en los mecanismos que dan lugar a este tipo de erosión, sobre todo en los aspectos de la intercepción de la precipitación antes de la infiltración o la modificación de la escorrentía superficial.

La cartografía, como elemento de delimitación de áreas vulnerables, tiene que integrar todas las variables que intervienen en estos procesos, pero fundamentalmente debe generarse a partir de bases cartográficas elementales que a escalas medias detallen con la mayor precisión posible los materiales detríticos, su basamento y las variaciones en los usos del suelo, aparte de las variables topográficas y climáticas.

\section{Conclusiones}

1. Las características del episodio pluviométrico que ha dado lugar al deslizamiento son bien distintas a las de otros episodios en los que las elevadas intensidades de lluvia provocaron una generalización de movimientos en masa en los Pirineos Orientales, muy ligados a procesos torrenciales (noviembre de 1982). En nuestro caso, si bien se produjeron otros movimientos en masa, el proceso aparece más aislado en el espacio.

Es evidente que el efecto detonante provocado por la sobrecarga del acuífero superficial, se originó a partir de un dilatado período de lluvias entre los meses de octubre a diciembre de 1996, prolongado hasta el 22 de enero de 1997, con un total de 737 mm de precipitación acumulada. El momento crítico de máxima humedad en el subsuelo se sitúa a partir de la fusión repentina del manto nival debido a un brusco ascenso de las temperaturas, fenómeno acompañado de precipitaciones líquidas moderadas.

2. El proceso descrito se compone de dos partes bien diferenciadas:

a) Un movimiento rotacional que afectó a un importante sector de la ladera.

b) Una colada de barro originada por los materiales acumulados en la cabecera de un torrente, por el cual discurrió de forma muy encajada.

Las dimensiones del deslizamiento se han adaptado a la morfología preexistente, fundamentalmente debido a las fuertes pendientes y al mar- 
cado cauce del torrente. Aun así, se ha originado un gran resalte a partir de la cicatriz de arranque que ha provocado el afloramiento del acuífero superficial, cuando antes lo hacía también en la cuenca colateral.

3. Las evidencias de otros movimientos de tierras en este sector se manifiestan en una serie de contrapendientes, como la existente en el campo de labranza situado en la zona de arranque del deslizamiento o en la parte superior de la ladera. Así mismo las referencias históricas atribuidas a problemas de estabilidad en el municipio de Cava, como las que obligaron al cambio de ubicación de éste, indican una cierta vulnerabilidad de este territorio. En este caso la toponimia y las referencias de los habitantes se muestran como una buena fuente de información de carácter prospectivo, más aun cuando en la cartografía de riesgos existente no se detectó esta zona como especialmente vulnerable (CLOTET et al., 1985).

Otro elemento de gran importancia es la naturaleza del substrato. Las facies garumnenses junto a unidades calizas afloran en numerosas localidades de los Prepirineos (TORAL, 1984) y han dado lugar en el pasado a los mayores deslizamientos de este sector de los Pirineos: Gòsol (1982) y Maçaners (1982) (CLOTET \& GALLART, 1983). La mayor frecuencia de procesos erosivos sobre estos materiales arcillosos indica una gran vulnerabilidad de las áreas que ocupan. El deslizamiento de Cava se encuentra entre los de mayores dimensiones del sector norte del Cadí; teniendo en cuenta la gran diversidad de materiales en esta zona y la limitada localización del Garumnense en esta vertiente ( 80 ha. aproximadamente) el movimiento de masa resulta especialmente significativo.

4. El abandono generalizado que experimentan estas zonas de montaña conlleva que este tipo de riesgos naturales no tengan el impacto social que podían tener antaño con una densidad de población mayor. Aun así, el desencadenamiento de estos procesos puede conducir al completo abandono del lugar si no se efectúan reparaciones urgentes de los daños materiales ${ }^{3}$. Por otro lado, la conservación de un medio montano eminentemente antropizado requiere una atención constante que ha ido decreciendo a lo largo del presente siglo. La «naturalización» de este espacio, que se expresa en cambios en la cobertura vegetal, el ciclo hidrológico, etc. juega un papel importante en la estabilidad de las laderas.

3 El deslizamiento afectó al depósito de aguas del pueblo, la línea eléctrica que suministra energía al barrio de Cal Pubill y la pista de acceso al mismo, así como la que se dirige al pie de la Sierra del Cadí. La reparación no se completó hasta un año después de los hechos, dejando sin electricidad durante este tiempo a una familia. 


\section{Referencias}

CLOTET, N. \& GALLART, F. (1983): Fenòmens d'esllavissaments esdevinguts durant els aiguats del Novembre de 1982 al Solsonès i Alt Berguedà: La Coma i Maçaners. Generalitat de Catalunya. Barcelona. Inédito.

CLOTET, N., GALLART, F., JULIÀ, R., MARQUÈS, Mª.A. \& SERRAT, D. (1985): "Mapa de riscs geomorfològics del Parc Natural del CadíMoixeró". Generalitat de Catalunya. D. G. Política Territorial. Barcelona. Inédito.

COROMINAS, J. (1984): Inestabilidad de laderas en el Pirineo Catalán. Tipología y causas. Jornadas de trabajo sobre inestabilidad de laderas en el Pirineo, Barcelona, Escola Tècnica Superior d'Enginyers de Camins, Canals i Ports, Universitat Politècnica de Catalunya, p. C.1-C. 53.

GARCÍA-RUIZ, J.M. \& PUIGDEFÁBREGAS, J. (1984): Inestabilidad de laderas en el Pirineo Aragonés: tipos de movimientos y su distribución geográfica. Jornadas de trabajo sobre inestabilidad de laderas en el Pirineo, Barcelona, Escola Tècnica Superior d'Enginyers de Camins, Canals i Ports, Universitat Politècnica de Catalunya, p. I.4.1.- I.4.12.

GARCÍA-RUIZ, J.M. \& LASANTA, T. (eds.) (1994): Efectos geomorfológicos del abandono de tierras. Sociedad Española de Geomorfología, Instituto Pirenaico de Ecología e Institución Fernando el Católico, 171 p., Zaragoza.

GARCÍA-RUIZ, J.M., WHITE, S.M., MARTÍ, C., VALERO, B., ERREA, M. P. \& GÓMEZ VILLAR, A. (1996): La catástrofe del Barranco de Arás (Biescas, Pirineo Aragonés) y su contexto espacio-temporal. C.S.I.C., Instituto Pirenaico de Ecología, 53 p., Zaragoza.

MARTÍN-VIDE, J. \& RASO, J.M. (1983): Marc atmosfèric. En Efectes geomorfològics dels aiguats de novembre de 1982. Informes 1, Barcelona, Pub. Servei Geològic de Catalunya, pp. 14-31.

MOLINA, D. (1996): Processos erosius als camps abandonats de Parc Natural del Cadí-Moixeró. Memoria de licenciatura. Universidad Autónoma de Barcelona.

SEOTERS, R. (1984): Fenómenos de inestabilidad de pendiente en la zona de Montanuy (provincia de Huesca). Jornadas de trabajo sobre inestabilidad de laderas en el Pirineo, Barcelona, Escola Tècnica Superior d'Enginyers de Camins, Canals i Ports, Universitat Politècnica de Catalunya, p. I.7.1I.7.7.

TORAL, T. (1984): Desplomes en los contactos Eoceno-Garumnense en la Conca de Tremp. Jornadas de trabajo sobre inestabilidad de laderas en el Pirineo, Barcelona, Escola Tècnica Superior d'Enginyers de Camins, Canals i Ports, Universitat Politècnica de Catalunya, p. I.8.1.-1.8.11. 


\section{PIRINEOS 149-150}

VIDAL, J. (1984): Problemas de inestabilidad de laderas en el Pirineo Central francés, relacionados con la ordenación del territorio. Jornadas de trabajo sobre inestabilidad de laderas en el Pirineo, Barcelona, Escola Tècnica Superior d'Enginyers de Camins, Canals i Ports, Universitat Politècnica de Catalunya, p. III.1.1-III.1.6. 